\title{
Design of MyServer: a Residential Server in Smart Home Systems
}

\author{
Wei-Chung Teng ${ }^{1}$, Yu-Chun $\mathrm{Pao}^{2}$, Sheng-Luen Chung ${ }^{2}$ \\ Department of Computer Science and Information Engineering ${ }^{1}$, \\ Department of Electrical Engineering ${ }^{2}$, \\ National Taiwan University of Science and Technology \\ weichung@mail.ntust.edu.tw, berylabalone@seed.net.twm,slchung@mail.ntust.edu.tw
}

\begin{abstract}
This paper addresses the design and implementtation of a residential gateway: MyServer, which is to provide services regarding home security, inventory tracking, facility management, and family care in a smart home system. Inherently an interactive and multitasking system driven by peripherals connected through wireless sensor networks, the overall MyServer architecture is designed with six clearly defined core service modules operating on a message oriented middleware (MOM) framework. Each of these modules is implemented with a programming template utilizing event publish/subscribe messaging mechanism. This paper also demonstrates a successful implementation of the proposed MyServer system in an emulated home environment, where peripherals are connected through ZigBee wireless sensor network.
\end{abstract}

\section{Introduction}

A smart home is an intelligent system composed of a wide variety of computerized devices. Via the integration of various technologies into a smart home system, it aims on improving the quality of life, saving energy through a monitoring system, and providing a convenient way of operating home devices [1][2]. Along with the continuous development of new technologies, it is predictable that more and more home devices are to be appended into a smart home system. Therefore, a successful smart home system should be able to dynamically append and configure various kinds of home devices, and to provide an integrated interface to the members of home.

To allow compatibility between different service providers, the design of a smart home in the commercial market needs to follow a given protocol in the development of various kinds of services. However, this paper emphasizes on enhancing the quality of households without considering entertainment for each family or commercial demand. The development of the smart home system uses a number of traditional technologies which allows it to be lightweight in nature. Directions of the design of our smart home system are as follows:

1) Integration platform: Building up an integration platform and a collaborative protocol to manage a variety of devices and services in the smart home.

2) Full functionality: developing an information dissemination management system for four applications to show detailed information about households.

3) Configurability: providing the configuration service to allow the system to meet the different requirements of each family.

\section{Technical Review}

In recent years, an increasing number of research groups sponsored by their government are entering the field of smart home technology with different approaches and focuses respectively. In addition, both industrial and academic communities have made a tremendous effort to address this issue. For instance, Microsoft research group have been developing EasyLiving [4] project since 1998.

Some efforts are reported to integrate wireless sensor networks (WSNs) with a smart home system. Yu et al. [5] proposed a cluster-based middleware architecture, which consists of a cluster control layer and a resource management layer, for the development of a virtual machine model in WSNs. In addition, Heinzelman et al. [6] developed a new middleware called MiLAN, which allows different applications to specify its management policy on controlling the network and sensors. 
In Greece, Nikolaidis et al. [7] investigated a promising solution for configuration and management in home networks, which is the CPE WAN Management Protocol (CWMP) developed by DSL Forum. Moreover, they combined it with the XMLbased messaging system to achieve the goal of automating remote configuration for home device, as well as presenting relevant tool suites covering the aspects they discussed [8]. Furthermore, in order to accelerate the deployment of new services, they proposed a new solution bridging the CWMP with UPnP-enabled appliances [9].

In the United Kingdom, Kolberg et al. [10] addressed compatibility issues between services in a home environment, as well as presented a devicecentric approach to detect and to avoid interactions between appliances which control networks based on an OSGi platform. In addition, they proposed a user interface which uses digital pen and paper to control networked appliances and is also integrated with OSGi [11]. Merabti et al. [12] discussed the current European research projects and initiatives within the area of residential gateway and networked appliances.

In Korea, there is one ongoing project, Ubiquitous Autonomic Computing and Network (uAuto) [13], which is to develop intelligent information processing for ubiquitous services, and to implement real ubiquitous environments for demonstration. The UTAGENT [14] design in this project uses case-based reasoning (CBR) to acquire knowledge about user actions. Moreover, Han et al. and Cho presented a new middleware architecture called HOMEROS [15], which adopted a hybrid-network model to efficiently manage enormous resources, context, location, and various services. In 2006, Kim et al. and Cho [16] proposed a new distributed clustering algorithm for wireless sensor network, providing a new method to reduce the cluster formation cost.

There are a number of studies which are based on OSGi (Open Service Gateway Initial). Choi et al. [17] proposed a context-aware middleware to be adopted as a home network framework, utilizing six basic data values for predicting and learning the user's preference for the home appliance. SangOk et al. [18] indicated the importance of implementing an initial provisioning function for home gateway. Lee et al. [19] and Hwang et al. [20] described the correlation between service delivery and management platforms, which is a major issue for individual companies providing ubiquitous home network services.

Additionally, ZigBee is becoming widely used in the control and communication of home appliances. Park et al. [21] combined ZigBee with IR to control household
CE (Consumer Electronics) by Z-URC (ZigBee based universal remote control) and Z2IR (ZigBee to infrared), as well as applying a novel method of using multiple timers to reduce power consumption of the ZigBee module. Furthermore, the mixture of ZigBee and UPnP (Universal Plug and Play) [22] has become an ideal solution to the problem of not being able to control home network appliances on the internet, providing an internet gateway for users to access local home network devices.

In Japan, Ishikawa et al. [23] indicated that COTS (Commercial off-the-shelf) software such as Java and Linux are suitable for implementing home appliances. They proposed a system coordinating various home appliances, called SENCHA [3], which is built on the OSGi component framework. The system can dynamically construct a dedicated user interface to suit various kinds of users' requirements.

In addition, Yamazaki et al. [24] proposed a home network service middleware called "UKARI-Kernel," which is used for distributed and collaborative networked appliances and sensors. In 2004, they built a real-life test bed [25], called the "Ubiquitous Home," for home context-aware service experiments, as well as presenting two experiment results to monitor living human activities. After completion of the test bed, they introduced various types of sensors to detect environmental information, and implemented contextaware service in their test bed [26]. Moreover, Yamazaki [27] raised an important issue of going beyond smart homes that automate all tasks for us. Researchers need to take into account this point when designing a smart home.

Besides these, there are still other architectures have been proposed. Igaki et al. [28] presented a serviceoriented framework for networked appliances to achieve both the evolution of HNS (home network systems) and the appliance interoperability. By these methods, it is helpful for non-expert users to develop integrated services easily. Furthermore, Nishimoto et al. [29] presented a digital rights management (DRM) system for digital broadcasting based on home servers (DBHS) using receivers with large-capacity storage devices. The main advantage is that the DRM system enables not only the control of content viewing at the time of reception of broadcast, but also the playback of stored content.

\section{The residential server}

MyHome system consists of a host component, called MyServer, which performs the message exchange and a remote component, called MyIcon, 
which provides a user interface for controlling home devices. In this section, the overall system architecture of MyHome is described.

\subsection{MyServer}

As Fig. 1 shows, we deploy a WSN technology Zigbee to collect and to integrate information about the supported four kinds of applications. MyHome Server (MyServer) architecture can be divided roughly into three parts with different interfaces. At first, home devices necessary to the four kinds of applications are associated with Zigbee Coordinator (ZC). These end devices are linked in a star topology centrally controlled at the coordinator, which is the central dispatcher managing the whole network communication. Secondly, the coordinator is connected to MyServer computer via a RS232 serial connector. Finally, the database on MyServer stores all kinds of data from home devices. Family members manage and access context-aware information in their living environment. Thus, based on the Zigbee/IEEE 802.15.4 standard [30], we build up an integration platform which consolidates information gathered from the four kinds of applications.

In addition, MyServer contains built-in features for alarm handling, including two notification methods: SMS and E-mail. If a house fire or other emergencies occur, MyServer will send a message to family members immediately.

\subsection{MyIcon}

As Fig. 1 shows, MyIcon is an interface for remote access to MyServer. In the top-left corner of the figure is MyServer, which set up at home; in the right-hand side is a MyHome icon for decision making and information integration on a remote computer. The icon is similar with the My Computer icon that is on a typical Windows environment desktop. When the user executes a double click, the hardware and software status of this computer will be displayed. Similar to the My Computer icon, the MyHome icon exhibits information on security, family, inventory, and facility. With the design pattern of MyIcon, family members would be able to amass an assortment of information about household activities at their finger tips. Therefore, with the assumption that there is an internet connection available and a computer with a USB flash drive which contains identification inserted into it, we will be able to maintain control under different household situation.

\subsection{Application Categories of MyHome}

In general, a smart home is concerned with linking up with entertainment-oriented services due to the development of these services by the IT (Information Technology) industry. However, we prefer to integrate a variety of information that encompasses the need of most families, including security, family member, inventory, and facility:

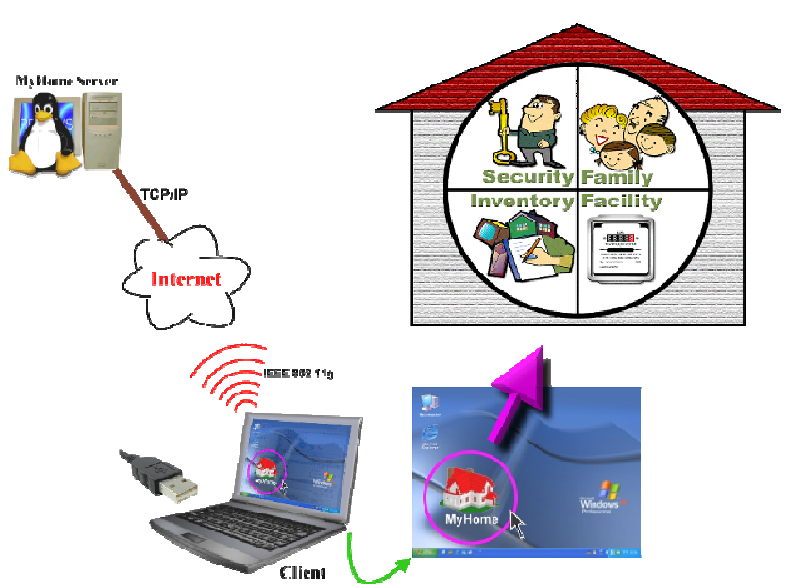

Figure 1. Remote access to MyHome via Mylcon interface

1. Security: It is important to enhance household security to suit the need of contemporary home life. To protect themselves against intruders, a general family would install a security system to ensure personal household security. However, they are charged an extra amount of monthly management fees by the Security Company. MyHome system combines security sensors with MyServer for centralized management, collects a variety of sensor status using Zigbee Wireless Network, and records them in the system. Through the happening times and locations of information dissemination service in MyIcon, family members obtain abnormal events from records.

2. Family: Health care is essential to ensure the fitness and well-being of every family member. For the modern individual with a busy lifestyle, he or she not only needs to monitor his or her physical condition but also learns how to keep his or her personal health in good condition. Furthermore, there is also a need to observe the health of other family members, especially elders who live alone. Therefore, the MyHome system links MyServer with devices which can measure basic physiological information, such as heart rate and blood pressure, etc. Based on the age and fitness levels of family members, we set a threshold values to monitor their physical condition. If our system is connected to the nearest hospital, family members would be able to 
obtain medical treatment from medical personnel in time when any abnormalities are detected.

3. Inventory: It is helpful for family members to construct a daily behavior monitoring system in the smart home. If our system records and displays family behavior information on different location context, we will have a household activity of member of a family in hand. Consequently, MyHome system uses RF (Radio Frequency) technology for building up a people location system, connecting ZED with RFID (Radiofrequency identification) reader for a bridge between MyServer and RFID. There will be detected whether a person is located in a given environment and showed the location information of people on the condition that family members wear an active RFID tag.

4. Facility: Energy conservation is an important initiative all over the world. For the family, monthly household utilities bills would be reduced if efficiency is improved and thus, energy wastage reduced. Of course, the environment would also benefit as less resources are wasted. Consequently, MyHome system utilizes the method of image recognition to record data on home meters such as the water meter, the gas meter, and the electricity meter, etc. Through these records, family members are able to monitor the condition of energy usage at home. In the event that the set quota of the day has been exceed. MyServer would

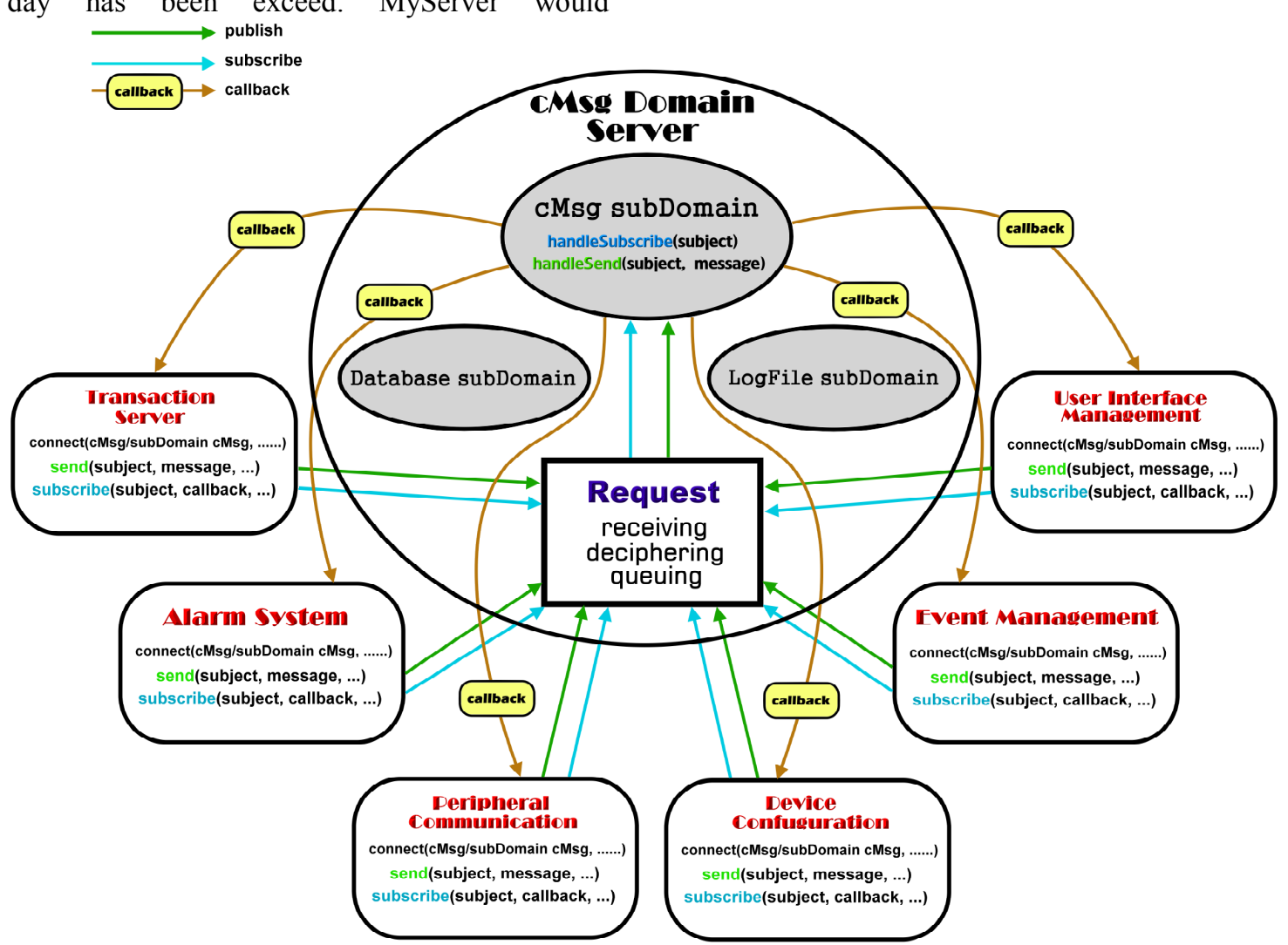

Figure 2. The cMsg domain server and the six modules implementing all functionality of MyServer automatically remind them to take note of the usage of energy at home constantly.

\section{System design of MyServer}

The design of an entire integration platform is composed of three major parts: a messaging system, entity-relationship model (ER model), and communication with ZigBee. The messaging system is needed for multi-processes to exchange information. The ER model is used in the building up of a database system, covering four categories of data sets. The communication with ZigBee uses a protocol to bridge the gap between MyServer and ZigBee Coordinator.

\subsection{The Messaging System: cMsg}

cMsg package [31] is a type of message oriented middleware (MOM) [32], supporting the exchange of general-purpose messages across distributed processes. It not only provides a full-featured asynchronous publish/subscribe messaging mechanism but also supports synchronous peer-to-peer messaging transmission. For asynchronous communication, a message published to cMsg domain can be delivered to any amount of subscribers in case they subscribe to the 
abstract subject. For synchronous communication, a published message is received by only one subscriber.

The central theme of cMsg is based on messaging space, called a domain, in the package. It is similar to the concept of Domain Name System (DNS) on the internet; cMsg defines a Universal Domain Locator (UDL) for specifying different domains and subdomains. Fig. 2 shows the cMsg domain server, which contains one section for receiving requests and three different sub-domains for handling various requests.

In the MyServer application, the whole integration platform is composed of six modules:

1. Peripheral Communication System for receiving all of the incoming event messages from ZigBee nodes.

2. Device Configuration System for ZigBee device initialization.

3. Transaction Server for accessing the database.

4. Event Management System for handling all of the incoming data.

5. Alarm System for the notification of abnormal events.

6. User Interface Management System for a bridge between MyServer and MyIcon.

We adopt the cMsg sub-domain to handle the exchange of messages between modules, and each module is constantly connected to the cMsg domain and receives messages from other modules all the time. In the cMsg domain, each connected process needs to include a callback function, which is to handle the received messages if the process subscribes to a certain subject. When a message has been published, the function executes a corresponding handler. The technique is a sort of event-driven programming [33], commonly being adopted for developing MOM.

\subsection{Peripherals connected through ZigBee}

There are many ways to communicate with ZigBee, such as through RS232, TCP/IP, and USB, etc. In this paper, we use a RS232 serial port to connect ZigBee with MyServer, as well as provide Peripheral Communication System for data transmission. Therefore, it is necessary to define a frame format of the data packet as a serial communication protocol in the MyHome system.

The Peripheral Communication System is responsible for communicating with ZigBee Coordinator (ZC) and continuously awaits $\mathrm{I} / \mathrm{O}$ for data transmission. It not only receives a data frame from $\mathrm{ZC}$ when collecting sensor data but also sends a data frame to $\mathrm{ZC}$ if users need to control the ZEDs.

For receiving data, the Peripheral Communication System checks the CRC algorithm to verify whether the transmission of the frame is error-free when a data frame is received. Next, it publishes this message with its subject as 'frame' and its type based on the four different applications. Finally, the Event Management System receives the payload of the frame and determines where to dispatch the frame.

For sending data, the Peripheral Communication System subscribes to the message whose subject is

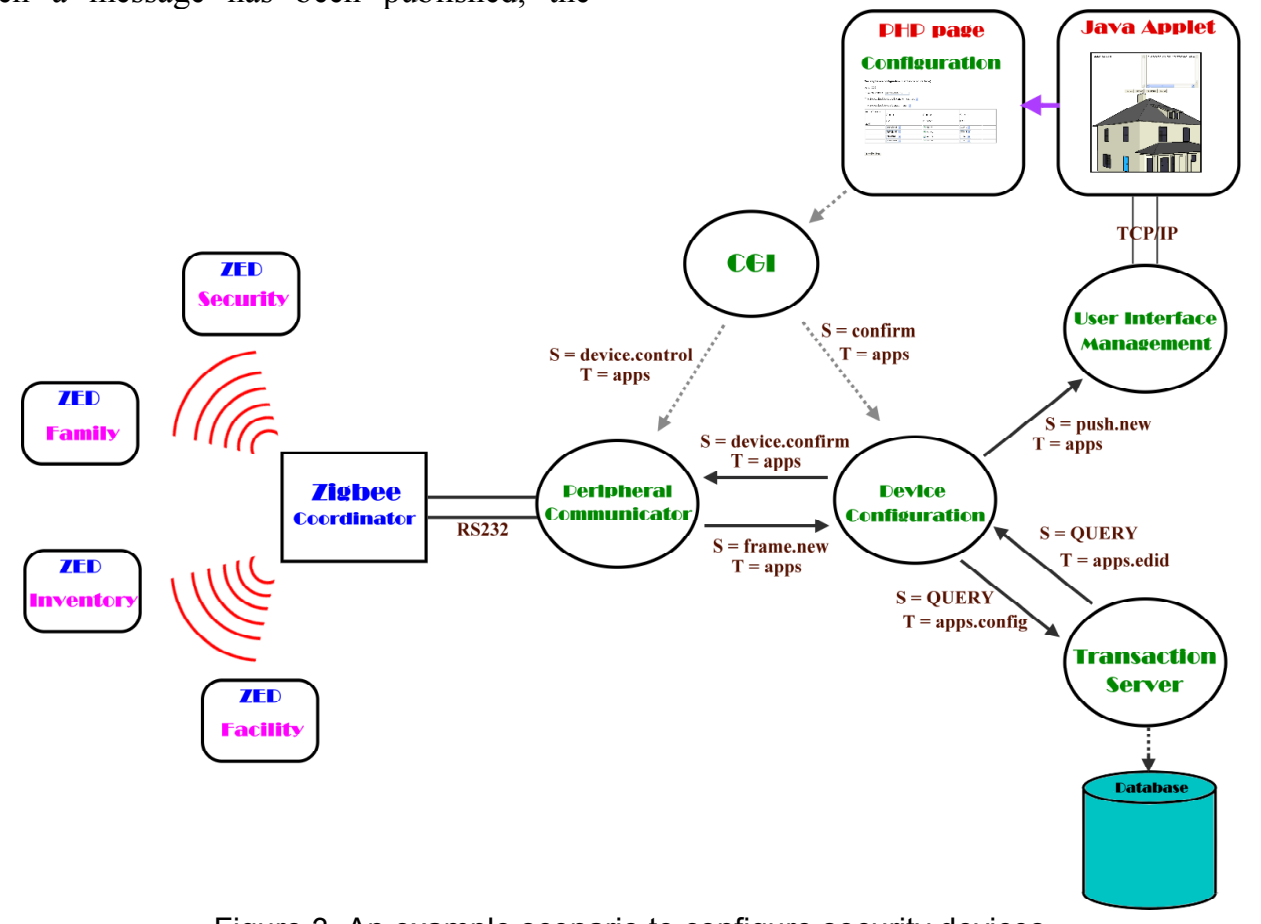

Figure 3. An example scenario to configure security devices 
related to 'device' after receiving control commands from users. If the user interface sends control signals to device, it will take the callback immediately and send a control frame to $\mathrm{ZC}$. ZED will receive the command and perform the corresponding task.

\section{Demonstration}

Now we explain the real process of MyHome system via an example scenario about how an alarm is generated by a triggered security sensor such as glass break sensors, reed switches, or fire detectors, etc. The whole process is illustrated in Fig. 3. In the first step, the security sensor, or the ZED, is automatically detected and is manually added by users from MyIcon interface. When a new device is connected at the first time, it is programmed to sends a data frame of fixed format to the Peripheral Communication System via the RS232 serial port. This frame includes the ID, the category of applications this device belongs, and other registration information. The Peripheral Communication System then decomposes the received frame into two parts: the ZED address field and the payload field, as well as publishes the ZED address to the cMsg domain. After that, the Device Configuration System obtains the ZED address from its callback function, and then dispatches it to the Security handle. Next, the Security handler publishes the stored procedure named add_new_ZED () contains the address to the cMsg domain. The Transaction Server will get the call from its callback function, and register the address in the MySQL database.

After registered the address in the database, the Transaction Server will obtain a ZED identification from the database, which is used to identify each ZigBee nodes in MyHome system through a different MAC address; then, it publishes the identification to the cMsg domain. Device Configuration System will get the identification from its callback function, and then publishes the identification with its subject as 'push.new' to the cMsg domain to notify the User Interface Management System that a new device has registered for the MyHome system. A configuration page for users to set the parameters of security sensors is automatically loaded when the User Interface Management System obtains the identification from its callback function.

After setting various parameters and clicking the submit button, a CGI program is invoked to connect to the cMsg domain and publish the identification with its subject as 'confirm' to notify the Device Configuration System that the configuration procedure is finished. The Device Configuration System will get the message from its callback function, and then publish the identification which has been registered in the cMsg domain.

After the Peripheral Communication System obtains the identification from its callback function, it sends a confirm frame notifying the security ZED of the successful completion of the configuration process. Finally, the Security ZED is invoked which starts to send the status of the security sensors.

\section{Conclusion}

In this paper, we proposed a novel integrated system which supports ZigBee nodes associated with various appliances to establish a smart home. The architecture is based on the cMsg messaging system, which provides extensibility to the entire system. By making use of the feature of device of ZigBee, many household sensor data can be integrated into the MyHome system.

The major contributions of this paper are to propose an event-driven, multi-threaded MyServer, composed of six core modules by a message oriented middleware, cMsg. When combined with an integrated database, MyServer provides the full functionality to dynamically append, remove, configure, and communicate with various kinds of home devices via ZigBee protocol, and thus supports the four categories of MyHome system services.

\section{References}

[1] D. Valtchev and I. Frankov, "Service Gateway Architecture for a Smart Home," IEEE Magazine on Communications, vol. 40, no. 4, pp.126-132, Apr. 2002.

[2] L. Jiang, D.-Y. Liu, and B. Yang, "Smart Home Research," in Proc. of 2004 International Conference on Machine Learning and Cybernetics, vol. 2, pp.659-663, 2629 Aug. 2004.

[3] H. Ishikawa, Y. Ogata, K. Adachi, and T. Nakajima, "Building Smart Appliance Integration Middleware on the OSGi Framework," in Proc. of the 7th IEEE International Symposium on Object-Oriented Real-Time Distributed Computing (ISORC'04), pp.139-146, 2004.

[4] S. Shafer, J. Krumm, B. Brumitt, B. Meyers, M. Czerwinski, and D. Robbins, "The New EasyLiving Project at Microsoft Research," in DARPA/NIST Workshop on Smart Spaces, July 1998.

[5] Y. Yu, B. Krishnamachari, and V.K. Prasanna, "Issues in Designing Middleware for Wireless Sensor Networks," IEEE Network, vol. 18, no. 1, pp.15-21, 2004.

[6] W.B. Heinzelman, A.L. Murphy, H.S. Carvalho, and M.A. Perillo, "Middleware to Support Sensor Network Applications," IEEE Network, vol. 18, no. 1, pp.6-14, 2004. [7] A. E. Nikolaidis, G. A. Doumenis, G. I. Stassinopoulos, M.-P. Drakos, and M. P. Anastasopoulos, "Management Traffic in Emerging Remote Configuration Mechanisms for 
Residential Gateways and Home Devices," IEEE Magazine on Communications, vol. 43, no. 5, pp.154-162, May 2005.

[8] A. E. Nikolaidis, S. S. Papastefanos, G. I. Stassinopoulos, M.-P. K. Drakos, and G. A. Doumenis, "Automating Remote Configuration Mechanisms for Home Devices," IEEE Trans. on Consumer Electronics, vol. 52, no. 2, May 2006.

[9] A. E. Nikolaidis, S. Papastefanos, G. A. Doumenis, G. I. Stassinopoulos, and M.-P. Drakos, "Local and Remote Management Integration for Flexible Service Provisioning to the Home," IEEE Magazine on Communications, vol. 45, no. 10, pp.130-138, Oct. 2007.

[10] M. Kolberg, E.H. Magill, and M. Wilson, "Compatibility Issues between Services Supporting Networked Appliances," IEEE Magazine on Communications, vol. 41, no. 11, pp.136-147, Nov. 2003.

[11] M. Kolberg and E.H. Magill, "Using pen and paper to control networked appliances," IEEE Magazine on Communications, vol. 44, no. 11, pp.148-154, Nov. 2006.

[12] M. Merabti, O. Abuelma'atti, and P. Fergus, "Networked Appliances and Home Networking," in Proc. of The 1st Int. Workshop on the Ubiquitous Home, Kyoto University, Japan, 2005.

[13] W.-D. Cho, S.-S. Kim, and H.-J. Yeh, "Introduction to the "uAuto" project - ubiquitous autonomic computing and network," in Proc. of the 2nd IEEE Workshop on Software Technologies for Future Embedded and Ubiquitous Systems, pp. 24-26, May 2004.

[14] N. Kushwaha, M. Kim, D. Y. Kim, and W.-D. Cho, “An intelligent agent for ubiquitous computing environments: smart home UT-AGENT," in Proc. of the 2nd IEEE Workshop on Software Technologies for Future Embedded and Ubiquitous Systems, pp.157-159, May 2004.

[15] S. W. Han, Y. B. Yoon, H. Y. Youn, and W.-D. Cho, "A new middleware architecture for ubiquitous computing environment," in Proc. of the 2nd IEEE Workshop on Software Technologies for Future Embedded and Ubiquitous Systems, pp.117-121, May 2004.

[16] D. Kim, S. Lee, and W.-D. Cho, "Energy efficient clustering routing algorithm for wireless sensor networks," Lecture Notes in Computer Science 4096 LNCS, pp.10781088, 2006.

[17] J. Choi, D. Shin, and D. Shin, "Research and Implementation of the Context-Aware Middleware for Controlling Home Appliances," IEEE Trans. on Consumer Electronics, vol. 51, no. 1, pp.301-306, Feb. 2005.

[18] S. Ok and H. J. Park, "Implementation of Initial Provisioning Function for Home Gateway Based on Open Service Gateway Initiative Platform," The 8th Int. Conf. Advanced Communication Technology ICACT 2006, vol. 3, pp.1517-1520, Feb. 2006.

[19] I.-W. Lee, H.-J. Park, K.-R. Park, and S.-H. Kim, “A Proposed Platform \& Performance Estimation of DigitalHome Service Delivery/Management Systems," in Proc. of the 3rd Int. Conf. on Information Technology: New Generations, pp.713-719, Apr. 2006.

[20] T. Hwang, H. Park, and J. W. Chung, "Design and implementation of the home service delivery and management system based on OSGi service platform," Int.
Conf. on Consumer Electronics (ICCE '06), pp.189-190, Jan. 2006.

[21] W.-K. Park, I. Han, and K.-R. Park, "ZigBee based Dynamic Control Scheme for Multiple Legacy IR Controllable Digital Consumer Devices," IEEE Trans. on Consumer Electronics, vol. 53, no. 1, pp.172-177, Feb. 2007. [22] K.-S. Kim, C. Park, K.-S. Seo, I.-Y. Chung, and J. Lee, "ZigBee and the UPnP Expansion for Home Network Electrical Appliance Control on the Internet," in Proc. of the 9th Int. Conf. on Advanced Communication Technology, vol. 3, pp. 1857-1860, Feb. 2007.

[23] H. Ishikawa, E. Tokunaga, and T. Nakajima, "A case study of implementing home appliance middleware on Linux and Java," in Proc. of the 2002 Symposium on Applications and the Internet (SAINT) Workshops, pp. 31-34, Feb. 2002.

[24] T. Yamazaki, D. Kwon, A. Sawada, and M. Minoh, "Emulator Implementation of Service Scenario Description Framework for Home Networked Appliances," in Proc. of the Joint Int. Conf. on Autonomic and Autonomous Systems and Int. Conf. on Networking and Services (ICAS/ICNS 2005), pp. 5-10, 2005.

[25] T. Yamazaki, "Ubiquitous Home: Real-life Testbed for Home Context-Aware Service," in Proc. of the 1st Int. Conf. on Testbeds and Research Infrastructures for the Development of Network and Communities, pp. 54-59, 2005. [26] T. Yamazaki, "Human Action Detection and Contextaware Service Implementation in a Real-life Living Space Test Bed," in Proc. of the 2nd Int. Conf. on Testbeds and Research Infrastructures for the Development of Networks and Communities, Mar. 2006.

[27] T. Yamazaki, "Beyond the Smart Home," in Proc. of 2006 Int. Conf. on Hybrid Information Technology, pp. 350355, 2006.

[28] H. Igaki, M. Nakamura, and K. Matsumoto, "A Serviceoriented Framework for Networked Appliances to Achieve Appliance Interoperability and Evolution in Home Network System," in Proc. of the 8th Int. Workshop on Principles of Software Evolution, pp.61-64, Sept. 2005.

[29] Y. Nishimoto, A. Baba, T. Kurioka, and S. Namba, "A Digital Rights Management System for Digital Broadcasting Based on Home Servers," IEEE Transactions on Broadcasting, vol. 52, no. 3, pp.167-172, Jun. 2006.

[30] 802.15.4-2003 IEEE Standard for Information Technology- Part 15.4: Wireless Medium Access Control (MAC) and Physical Layer (PHY) specification for Low Rate Wireless Personal Area Networks (LR-WPANS), 2003.

[31] E. Wolin, D. Abbott, V. Gurjyan, E. Jastrzembski, D. Lawrence, and C. Timmer, "cMsg - A publish/subscribe package for real-time and online control systems," 14th IEEE-NPSS Real Time Conference, no. 1547431, pp. 74-78, 2005.

[32] L. Fiege, M. Cilia, G. Muhl, and A. Buchmann, "Publish-Subscribe Grows Up: support for management, visibility control, and heterogeneity," IEEE Internet Computing, vol. 10, no. 1, pp.48-55, Jun.-Feb. 2006.

[33] S. Ferg, "Event-Driven Programming: Introduction, Tutorial, History," [Online]. Available: http://eventdrivenpgm.sourceforge.net/ 